\title{
STAT3 targeting by an aptamer-based conjugate for glioblastoma multiforme therapy
}

\author{
C. L. Esposito ${ }^{1}$, S. Nuzzo ${ }^{2}$, M. L. Ibba ${ }^{3}$, I. P. Grinev ${ }^{6}$, A. Gorbushin ${ }^{6}$, D. Grek ${ }^{6}$, I. Voronkovskii', O. S. Kolovskaya ${ }^{6,7}$, T. N. Zamay ${ }^{6}$, E. Morozov', \\ A. Koshmanova ${ }^{8}$, A. A. Narodov ${ }^{6}$, V. A. Khorzhevskii', E. E. Erakhtin ${ }^{6}$, A. V. Krat ${ }^{6}$, A. Yakovlev', P. A. Shesternya ${ }^{6}$, A. S. Kichkailo $0^{6,7}$, Gerolama Condorelli ${ }^{3,4}$, \\ Silvia Catuogno ${ }^{1}$, Vittorio de Franciscis ${ }^{1,5}$ \\ ${ }^{1}$ Istitute for Experimental Endocrinology and Oncology, Research National Council (CNR), Naples 80145, Italy \\ ${ }^{2}$ IRCCS SDN (Istituto di Ricovero e Cura a Carattere Scientifico, SYNLAB istituto di Diagnostica Nucleare), Naples 80143, Italy \\ ${ }^{3}$ Department of Molecular Medicine and Medical Biotechnology, "Federico II" University of Naples, Naples 80131, Italy \\ ${ }^{4}$ IRCCS Neuromed (Istituto di Ricovero e Cura a Carattere Scientifico Neuromed) Istituto Neurologico Mediterraneo, Pozzilli 86077, Italy \\ ${ }^{5}$ Institute of Genetic and Biomedical Research (IRGB), Research National Council (CNR), Milan, Italy \\ ${ }^{6}$ Professor V. F. Voino-Yasenetsky Krasnoyarsk State Medical University, Krasnoyarsk 660022, Russian Federation \\ ${ }^{7}$ Federal Research Center "Krasnoyarsk Science Center of the Siberian Branch of the Russian Academy of Sciences”, Krasnoyarsk 660036, Russian \\ Federation \\ ${ }^{8}$ Siberian Federal University, Krasnoyarsk 660041, Russian Federation
}

Conflict of interest. The authors declare the absence of obvious and potential conflicts of interest associated with the publication of this article. Citation: Esposito CL, Nuzzo S, Ibba ML, Grinev IP, Gorbushin A, Grek D, Voronkovskii I, Kolovskaya OS, Zamay TN, Morozov E, Koshmanova A, Narodov AA, Khorzhevskii VA, Erakhtin EE, Krat AV, Yakovlev A, Shesternya PA, Kichkailo AS, Condorelli G, Catuogno S, de Franciscis V. STAT3 targeting by an aptamer-based conjugate for glioblastoma multiforme therapy. Siberian Medical Review. 2021; (2):72-73. DOI: 10.20333/2500136-2021-2-72-73

Among brain cancers, glioblastoma multiforme (GBM) is the most common in adults, the most lethal, and the hardest to treat with a median survival of approximately 10 months. Disease relapse rate is very high and usually progressive disease rapidly evolve and patient succumb in few months. Surgical resection, when possible, followed by temozolomide chemotherapy associated with radiotherapy is the current state of care for patient suffering of GBM, however in patients undergoing relapse standard therapy has minimal benefices [1]. An important drawback in GBM treatment is that conventional therapies poorly affect a small population of stem-like cancer cells (glioblastoma stem cells, GSCs) that remain capable of repopulating the tumor. Thus, the development of strategies able to target GSCs represents an important challenge in oncology to effectively render the tumors unable to maintain themselves or grow. Nucleic acid therapeutics, including siRNAs and miRNAs/antimiRs, are emerging highly promising molecules for the precise treatment of refractory aggressive GBM. As matter of fact, transcription factors and micro-RNAs essential to maintain the tumor initiating capacity of GSCs and the ability to invade the normal brain have been described as potential therapeutic targets (including signal transducer and activator of transcription-3, STAT3, and miR-10b). However, the need for precise and safe targeted cell delivery and the lack of effective drugs to eradicate GSCs has revealed this as a challenging objective. In the last decade due to their high affinity for specific ligands, high intra-tumor penetration and chemical flexibility, short single-stranded nucleic acid aptamers are emerging as very attractive carriers for various therapeutic oligonucleotides $[2,3]$.

In order to develop a combined strategy enabling to target both the stem-like and the adherent bulk tumor cells, as targeting moieties to drive RNA drugs here we used two aptamers ligands for two receptor tyrosine kinase (RTKs) in combination. The first, named Gint4.T that binds to and antagonizes the RTK, PDGFR $\beta$, overexpressed in GSCs; the second, named GL21.T that binds to and antagonizes the RTK, AXL, overexpressed and involved in several tumor types including GBM. STAT3 has been reported as key regulator of the highly aggressive mesenchymal GBM subtype and of survival and propagation of GSCs. Using the Gint4.T aptamer, we recently designed a novel aptamer-siRNA chimera (AsiC, Gint4.TSTAT3). Because of the pivotal role of STAT3 in maintaining the tumor initiating capacity of GSC population and tumor relapse, here we explored the potential of Gint4.TSTAT3 to inhibit the STAT3-dependent gene expression impairing the stem-like GSCs phenotype. Further, we show that Gint4.T-STAT3 synergizes in vitro and in vivo with a chimera containing the anti-Axl aptamer GL21.T linked to the single chain antagonist of miR-10b (GL21.T10b) to effectively and specifically prevent patient-derived GSC function and expansion.

The biological effect of both chimeras Gint4.T-STAT3 and GL21.T-10b was discovered in vivo on immunosuppressed mice with xenotransplanted human glioblastoma. Primary cell cultures were obtained from the verified GBM tumor tissues taken immediately after the surgery the 
patient. Two neurospheres approximately $2 \mathrm{~mm}$ in diameter in $5 \mu$ l of GrowDex/DMEM media (1:1) were transplanted stereotaxically into the right brains of mice with one week immunosuppression (ciclosporin, ketoconazole, and cyclophosphamide). MRI and histological analyses proved the growth of glial tumors in mice brains. The treatment started on day 21 after tumor transplantation. Gint4.T-STAT3; GL21.T-10b; Gint4.T; GL21.T; scrambled aptamer, were injected intraperitoneally. Not treated mice were used for negative control. Mice were treated on days $21,22,23,24,26,28$ after tumor transplantation. The course of treatment was controlled using MRI on day 1,6 , and 9 of treatment (days 21,26, 29 after tumor transplantation). On day nine after the final MRI mice the brains were collected for histological immunohistochemical RNA expression analyses.

MRI preliminary results demonstrate that treatment with Gint4.T-STAT3 and GL21.T-10b of mice GBMs increased the number of necrotic or swelling zones in tumor area in the mice brains, at the same time not treated gliomas were sufficiently bigger in size and with less necrosis.

Our results provide the rationale for the development of effective AsiC-based therapies showing the great potential to allow a complete GBM tumor eradication and improve patient outcomes $[4,5,6]$.

\section{References}

1. Taylor OG, Brzozowski JS Skelding KA. Glioblastoma Multiforme: An Overview of Emerging Therapeutic Targets. Frontiers in Oncology. 2019;(9);963.

2. Carro MS, Lim WK, Alvarez MJ, Bollo RJ, Zhao X, Snyder EY. The transcriptional network for mesenchymal transformation of brain tumours. Nature. 2010;(463): 318-325.

3. Kwiatkowska A, Symons M. Signaling determinants of glioma cell invasion. Advances in Experimental Medicine and Biology. 2013;(986):121-141.

4. Esposito CL, Catuogno S, Condorelli G, Ungaro P, de Franciscis V. Aptamer Chimeras for Therapeutic Delivery: The Challenging Perspectives. Genes (Basel). 2018;(9):11.
5. Esposito CL, Nuzzo S, Catuogno S, Romano S, de Nigris F, de Franciscis V. STAT3 Gene Silencing by Aptamer-siRNA Chimera as Selective Therapeutic for Glioblastoma. Molecular Therapy - Nucleic Acids. 2018;(10):398-411.

6. Esposito CL, Nuzzo S, Ibba ML, Ricci-Vitiani L, Pallini R, Condorelli G, Catuogno S, de Franciscis V. Combined targeting of glioblastoma stem-like cells by neutralizing RNA-bio-drugs for STAT3. Cancers. 2020;12(6):1434.

\section{Author information}

Carla L. Esposito, PhD, Istituto di Endocrinologia ed Oncologia Sperimentale, Consiglio Nazionale delle Ricerche (CNR), 80100, Naples, Italy; e-mail: c.esposito@ieos.cnr.it.

Silvia Nuzzo, PhD, IRCCS SDN, 80100, Naples, Italy.

Michael Ibba, researcher, Department of Molecular Medicine and Medical Biotechnology, "Federico II" University of Naples, 80131 Naples, Italy.

Igor Grinev, doctor, Professor V. F. Voino-Yasenetsky Krasnoyarsk State Medical University, Krasnoyarsk 660022, Russian Federation.

Anton Gorbushin, student, Professor V. F. Voino-Yasenetsky Krasnoyarsk State Medical University, Krasnoyarsk 660022, Russian Federation.

Daniil Grek, student, Professor V. F. Voino-Yasenetsky Krasnoyarsk State Medical University, Krasnoyarsk 660022, Russian Federation.

Ivan Voronkovskii, student, Professor V. F. Voino-Yasenetsky Krasnoyarsk State Medical University, Krasnoyarsk 660022, Russian Federation.

Olga Kolovskaya, PhD, Professor V. F. Voino-Yasenetsky Krasnoyarsk State Medical University, Krasnoyarsk 660022, Russian Federation; Federal Research Center "Krasnoyarsk Science Center of the Siberian Branch of the Russian Academy of Sciences", Krasnoyarsk 660036, Russian Federation.

Tatiana Zamay, Professor, Professor V. F. Voino-Yasenetsky Krasnoyarsk State Medical University, Krasnoyarsk 660022, Russian Federation.

Evgeniy Morozov, PhD, Federal Research Center "Krasnoyarsk Science Center of the Siberian Branch of the Russian Academy of Sciences", Krasnoyarsk 660036, Russian Federation. Anastasia Koshmanova, student, Siberian Federal University, Krasnoyarsk 660041, Russian Federation.

Andrey Narodov, Professor, Professor V. F. Voino-Yasenetsky Krasnoyarsk State Medical University, Krasnoyarsk 660022, Russian Federation.

Vladimir Khorzhevskii, PhD, Professor V. F. Voino-Yasenetsky Krasnoyarsk State Medical University, Krasnoyarsk 660022, Russian Federation.

Evgeniy Erakhtin, doctor, Professor V. F. Voino-Yasenetsky Krasnoyarsk State Medical University, Krasnoyarsk 660022, Russian Federation.

Alexey Krat, PhD, Professor V. F. Voino-Yasenetsky Krasnoyarsk State Medical University, Krasnoyarsk 660022, Russian Federation.

16.Artem Yakovlev, student, Siberian Federal University, Krasnoyarsk 660041, Russian Federation.

Pavel Shesternya, Professor, Professor V. F. Voino-Yasenetsky Krasnoyarsk State Medical University, Krasnoyarsk 660022, Russian Federation.

Anna Kichkailo, PhD, Professor V. F. Voino-Yasenetsky Krasnoyarsk State Medical University, Krasnoyarsk 660022, Russian Federation; Federal Research Center "Krasnoyarsk Science Center of the Siberian Branch of the Russian Academy of Sciences", Krasnoyarsk 660036, Russian Federation.

Gerolama Condorelli, Professor, Institute of Genetic and Biomedical Research (IRGB), Research National Council (CNR), Milan, Italy.

Silvia Catuogno, PhD, Istitute for Experimental Endocrinology and Oncology, Research National Council (CNR), 80145 Naples, Italy.

Vittorio de Franciscis, Professor, Institute of Genetic and Biomedical Research (IRGB), Research National Council (CNR), Milan, Italy; Istitute for Experimental Endocrinology and Oncology, Research National Council (CNR), 80145 Naples, Italy.

Received 18 February 202 Revision Received 18 March 2021 Accepted 31 March 2021 